\title{
Application of the Familiarization, Functionality and Testing Design Method
}

\author{
Jon Keech", Rod Fauvel", Clif Johnston*, Greg Kawchuk ${ }^{* *}$ \\ *Department of Mechanical Engineering, University of Calgary \\ ${ }^{* *}$ Faculty of Kinesiology, University of Calgary \\ jekeech@ucalgary.ca
}

\begin{abstract}
Low back pain $(L B P)$ is a major societal burden as it is exceedingly common and extraordinarily difficult for clinicians to diagnose. New methods of diagnosis are required if meaningful strategies related to prevention and treatment are to be found.

A new design technique known as Familiarization, Functionality and Testing (FFT) was applied in order to develop a solution. This functionality driven technique is well suited to problems where the designer is unfamiliar with the area of design. Through the application of this method, the designer was able to generate solutions to the problems that satisfied customer demands.
\end{abstract}

\section{Introduction}

Low back pain (LBP) is an increasingly common and expensive societal burden. The lifetime risk of incidence for adults is $80 \%$ with a total of 25.9 million adults in the US experiencing some form of LBP in the year 1998 [1]. The spine is extraordinarily complex resulting in $85 \%$ of spinal complaints being improperly diagnosed by practitioners [2]. These factors contributed to an overall health care cost of $\$ 90.7$ billion US in 1998 [3]. If the number of correct diagnoses were improved by a mere $1 \%$, a potential health care savings of nearly $\$ 1$ billion yearly could be realised.

Research points to a strong link between an increase in spinal stiffness and low back pain [4]. If practitioners could reliably measure spinal stiffness in vivo, they would be better poised to correctly diagnose spinal complaints. Unfortunately, current diagnostic technologies are inadequate for the purposes of practitioners. Therefore, new diagnostic technologies must be developed if meaningful solutions to the problem of diagnosing and treating LBP are to be found [5].

Practitioners require a system that is affordable, easy to use and yields measurements that are both accurate and reliable. As the system is to be used on the general population, diagnosis must also be non-invasive, pain free and cause no long-term ill health effects in order to remain an attractive option in a competitive marketplace.

This project presented several unique challenges to the designer, the greatest of which was the tight project timeline. For an experienced designer familiar with the area of design this could be overcome without a great deal of difficulty. However, the designer on this project was a novice with little knowledge of the area of design and project background. This produced several problems that would require innovative and timely solutions. The most notable of these solutions was the implementation of the Familiarization, Functionality and Testing design method.

\section{Background}

Practitioners use many techniques to diagnose LBP; the majority of these rely on the subjective measurement of spinal stiffness. Hawk et al. have found that subjective techniques such as manual palpation yield little consensus between practitioners [6]. It was found that inexperienced examiners were more likely to diagnose a greater number of subluxations in pain free patients than their more experienced counterparts. This would by extension lead to a greater number of patients seeking treatment unnecessarily and in turn an increase in cost to the health care system without increasing the effectiveness of treatment. Hawk et al. suggest a need for new techniques that improve the interexaminer reliability of current manual techniques if diagnosis rates are to be improved but do not discount the potential of current manual techniques when used in conjunction with objective measures.

There are many technological alternatives employed by practitioners and researchers that provide an objective compliment to manual palpation. However, each of these systems possesses limitations that render it unsuitable for our needs. The Roëntgen Stereophotogrammetric Analysis (RSA) system most closely approaches a legitimate solution and is currently considered the gold standard for accuracy for in vivo measurements. Unfortunately, this system is highly invasive, subjects patients to ionizing radiation and requires extensive calibration and computation to yield accurate results [7]. While RSA fails as a practical solution for a large clinical 
population, this system will be used as an accuracy benchmark upon completion of our own solution.

In the search for an imaging technology that could provide an accurate and safe method of imaging subcutaneous targets, both A-mode (Amplitude Modulation) and B-mode (Brightness Modulation) ultrasound [8] were investigated. Ultrasound solves many problems inherent in other technologies while introducing few tradeoffs. It allows practitioners to 'view' their target in real time during measurements, it provides a safe imaging medium with no long-term ill health effects and it is inexpensive relative to other imaging systems.

However, attenuation in tissue is a major problem with high frequency ultrasound. This effectively limits the frequency of transducers to below $5 \mathrm{MHz}$ when imaging of deep targets, such as transverse processes, is necessary [9]. As a result we are limited in the maximum practical resolution to a level below that of the RSA preferable over B-mode (Figure 2) for imaging deep targets as B-mode is constrained by a finite number of pixels and screen resolution. In essence a deep target will image identically to a shallow target when employing Amode ultrasound.

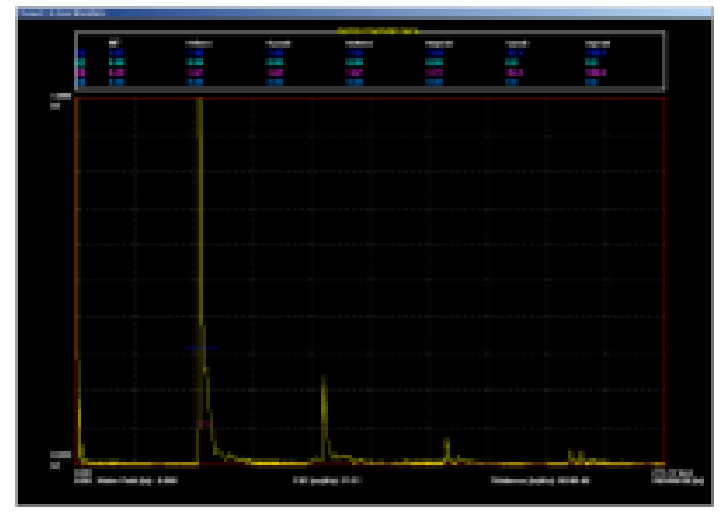

Figure 1 - A-mode Ultrasound Output

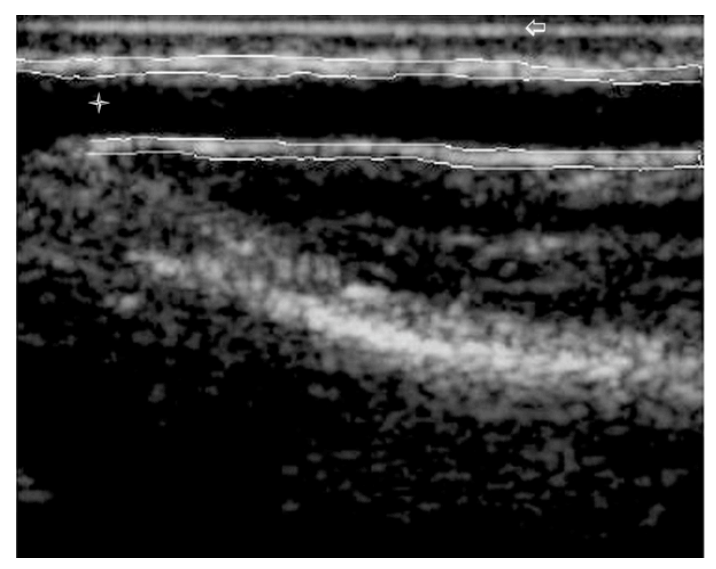

Figure 2 - B-mode Ultrasound Output
At the theoretical limit, the highest axial resolution that can be obtained with a $5 \mathrm{Mhz} \mathrm{A}$-mode transducer in tissue is $77 \mathrm{~mm}$. While not as accurate as RSA, it has been determined that ultrasound can provide an acceptable resolution level for accurately quantifying segmental motion in vivo.

The stiffness of the spine is determined much in the same manner as the stiffness of a spring is determined. A known load F is applied to the patient's lumbar spine; the resulting deflection is measured through a combination of the ultrasound based depth of the target TVP and the surface tissue deflection where . Using the relation we can determine the spinal stiffness $\mathrm{k}$.

By determining the stiffness of a patient's spine immediately after injury, there exists a baseline measurement that can be used to track a patient's recovery. If after a period of treatment the patient's spinal stiffness shows no signs of improvement, the practitioner can prescribe an alternate treatment modality. This gives practitioners a practical means of tracking the effectiveness of different treatments.

\section{Method}

A systematic design method similar to that described by Pahl and Beitz [10] was employed. Systematic methods lead the designer along a prescribed path during the determination of an acceptable solution. For many designers and design problems this is desirable. When a designer has completed a similar project (i.e. repetitive design) they may wish to simply follow a checklist to a solution. Systematic methods allow for this. When the project requires a new solution or the designer is new to the area of design (i.e. creative design) a checklist may not yield a solution quickly or at all. In these circumstances an alternate method must be employed.

During the initial stages of the project, research was conducted into the physics of ultrasound, the use of ultrasound in medicine, chiropractic manipulations as well as general background research and preliminary testing. Input from the client and other stakeholders was gathered and used to create a list of specifications. Based on these specifications, three functionally distinct preliminary concepts were generated for evaluation and testing. These concepts included: an acoustic mirror to alter the angle of the ultrasound (Figure 3), altering the angle of the transducer itself (Figure 4) and a rotating disc to translate the transducer between adjacent vertebrae (Figure 5). 


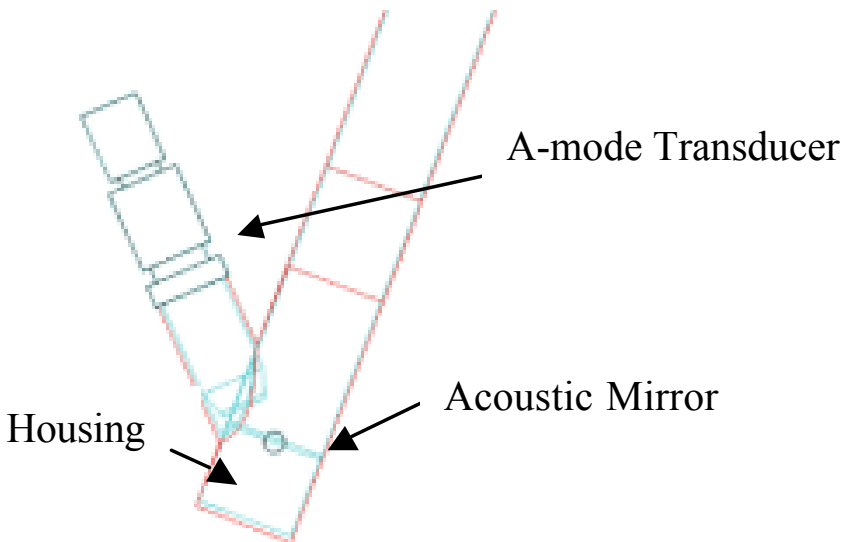

Figure 3 - Acoustic Mirror Model

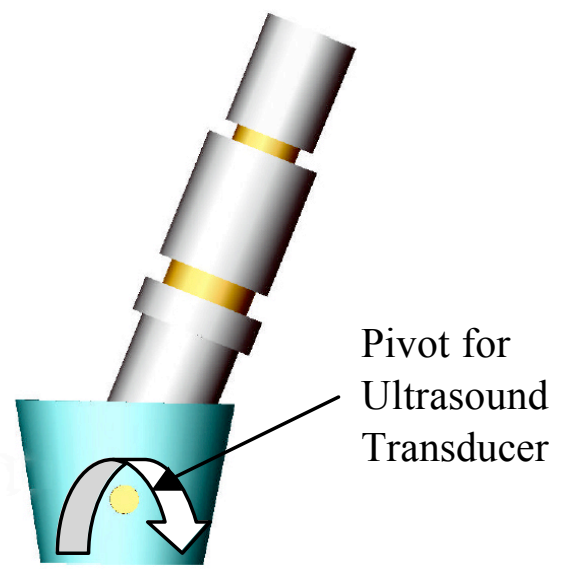

Figure 4 - Transducer Tilt Model

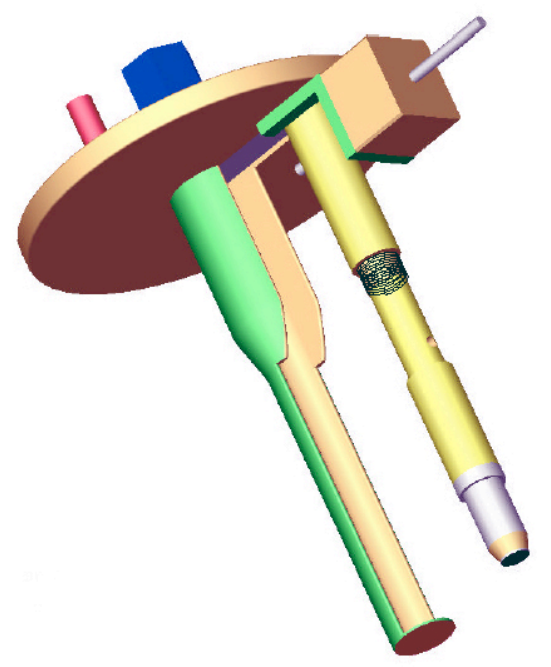

Figure 5 - Preliminary Rotating Disc Model

After these preliminary concepts were generated, proof of concept testing was conducted using phantoms of known dimensions. These tests demonstrated the capability of the acoustic mirror to accurately steer the ultrasound beam, however; minor attenuation was present in this system. It was also demonstrated that altering the angle of the ultrasound transducer itself would allow for the imaging of neighbouring TVPs. Further testing conducted on cadaveric porcine specimens demonstrated the ability of the rotating disc model to image multiple TVPs in rapid succession while pointing to the potential weakness of the other systems.

A design review was conducted to gather additional customer input and assess the project status. It was determined during the course of the review that the client deemed the proposed concepts acceptable for further development and that the project should continue to the next phase of concept development and refinement.

During this next phase, concepts from the initial concept stage were refined to the point where the customer and designer could more fully evaluate their potential functionality. This phase of the solution development required a great deal of designer labour and time as three independent solutions needed to be created. With the project deadline looming, the designer sought an alternative design method that would aid in developing a solution more rapidly.

Work during the year as a design coach with the first year engineering design class had introduced the designer to the Familiarization, Functionality and Testing design method [11]. This method (also known as FFT and The Design Trinity) relies on the completion of interrelated design activities rather than documentation to further the project. This method avoids a rigid and path oriented nature by identifying three broad design areas rather than a succession of smaller steps. The designer is given a greater number of potential solution paths as they move from area to area as dictated by the problem, not the design method. This improves the likelihood that a solution that fulfills the problem criteria is developed as well as improving the likelihood that the solution will be produced more quickly than through systematic means. If the process has become stalled at any point in the process, the designer need only shift to another area and complete another activity rather than forcing the project to fit the method. While other design methods include all the components of the FFT method, special emphasis is placed on them in this method. The familiarization component may be revisited throughout the course of a project by a designer and the testing area may be the first undertaken. The major shift from systematic approaches lies in the free form that application of the technique may take. There is no order prescribed by the method, it is up to the designer to determine the optimal implementation of the method based on the problem.

Having seen this method employed successfully by students with no design engineering background on creative design projects, the author chose to apply this method to his own project. The first step undertaken was an evaluation of the work completed to date. This included assessing the project definition and scope, the project specifications, the constraints and the worthiness 
of the preliminary concepts. Further research was conducted and it was deemed that the project definition and scope had been defined correctly but the functionality of the solution was ill defined. Upon updating the functionality definition, further testing showed that only one of the three concepts developed would be able to fulfill the functionality constraints, the linear translation model.

During this process, concept development was suspended. To an outsider this process may have appeared counter-productive as no 'real' design work was being done. This is one of the major differences between the FFT method and systematic methods. In the FFT method a great deal more time is spent on the project definition stage of the design to ensure that work completed during the embodiment stage does not require redoing at a later stage. This can be attributed to simple planning. If a sound plan is in place before the major 'design' is undertaken, there is less need for last minute panicking than if major components are left to chance.

Once this evaluation was complete, work was begun to fully define the required functionality of the solution. By focusing on the functionality rather than the form, solutions produced are no longer constrained by preconceived notions. That is, the designer is no longer thinking of what the solution must look like. They are now focused on what the solution must do. By severing the tie to form in the designer's mind we can be more certain that our solution will fulfill the client's desired functional requirements. This stage may be more difficult for the client than the designer as oftentimes the client will have a strong idea of what the solution will look like. It is the designer's responsibility to guide the client through the process of divorcing function from form and demonstrate to the client the capability of their solution to perform the required function.

Another challenge faced by the designer is that of determining the true problem requiring solution. For the majority of cases, the client approaches a design engineer to solve a symptom, not a cause [12]. Many systematic approaches view the problem definition more as a formality than a step in the process. For this reason the familiarization component of the FFT method is extremely valuable. During the familiarization aspects of design, the designer will gather information from the client and other stakeholders as well as interviewing outside parties in order to gain as broad a background as possible. This process aids the designer in uncovering deeper problems (and possibly the root of the problems) that the client may not have discovered. In this specific case, it was determined by the designer after a period of familiarization, that the problem was properly defined and the project could continue.

From a functionality standpoint, the indenter was very simple. It consisted of three main functional requirements. It must: apply a point load of known magnitude to a target TVP, image the target TVP and neighbours while sustaining the load and measure the indentation of the skin surface. Based on this functional assessment, modifications were made to the rotating disc concept and further testing was completed. An additional transducer was added to allow for the imaging of multiple TVPs simultaneously as it was determined through familiarization and testing that using a single transducer would not be sufficient for our purposes. These refinements lead to the first prototype, shown in CAD format in Figure 6.

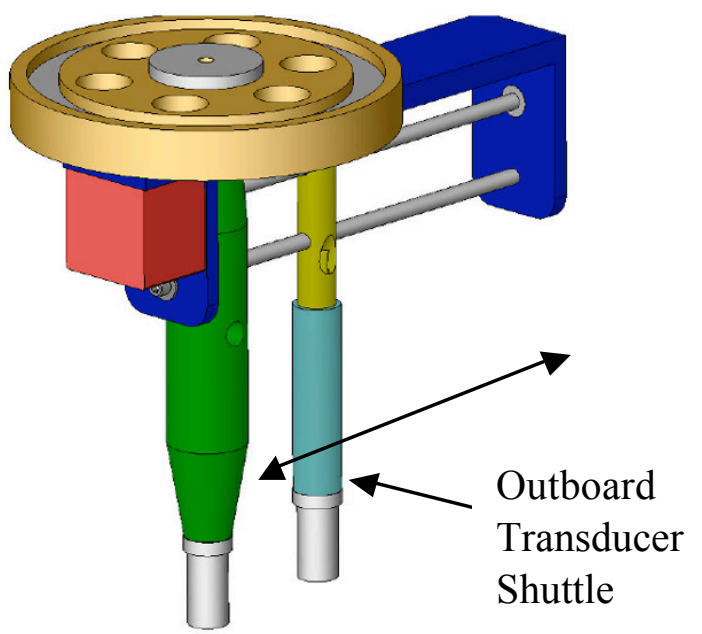

\section{Figure 6 - Prototype of Rotating Disc Model}

Upon manufacture of this prototype, testing was undertaken to discover any functionality shortcomings. These tests determined that the outboard transducer shuttle would be required to measure the deflection of the skin surface on the neighbouring image sites and would therefore need to be redesigned. Figure 7 shows the updated transducer shuttle that employs a Linear Variable Differential Transducer (LVDT) to measure the deflection on the skin's surface while Figure 8 shows an image of the fully assembled and revised rotational model.

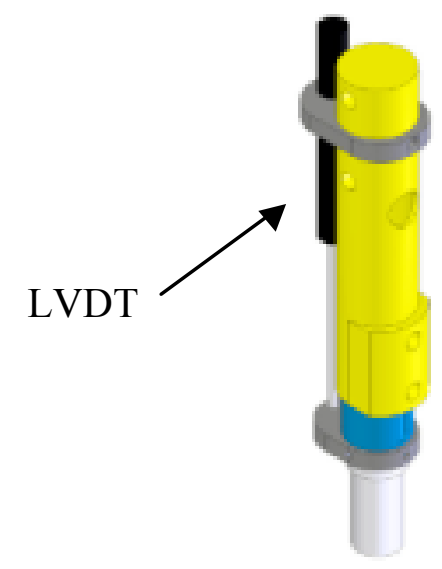

Figure 7 - Revised Shuttle Assembly 


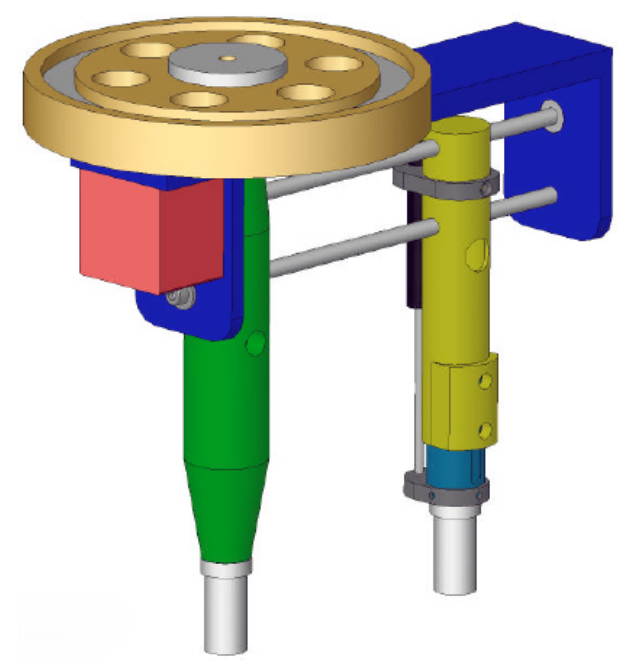

\section{Figure 8 - Revised Rotating Disc Model}

Preliminary testing indicates that this new solution meets customer demands and will provide an accurate method for quantifying spinal stiffness. Benchmarking against the RSA system is scheduled for completion within the next month.

\section{Discussion}

The contrast between the systematic approach and the FFT approach is similar to the contrast between the assembly line manufacturing process and the job shop manufacturing process. When a plant manufactures a single product repeatedly, having a highly systematic and repetitive process is desirable. With little change expected, there is little need for a flexible process and opportunities for streamlining exist. The products manufactured on an assembly line can be produced quickly and cheaply once the line has been set up. The initial assembly line setup and any changes necessary are both costly and time consuming making the assembly line manufacturing process unsuitable for many products.

A job shop is set up such that it can handle a relatively large number of smaller, independent jobs concurrently. It must remain flexible enough to handle a broad range of customer demands yet be efficient enough to turn a profit. In general, products manufactured in a job shop will be more costly than those manufactured on an assembly line but may also be more complex than assembly line products. This is extremely advantageous when manufacturing prototypes as this is an area where the requirement for modifications is much higher than when manufacturing tried and true products.

We can liken creative design challenges to prototype manufacture. We are given a new and previously unsolved problem and we must use our experience and creative ability to develop a means of developing a solution. It would not be logical to manufacture a prototype on an assembly line. If mistakes had been made the process would need to be stopped while the problem was determined and the necessary changes were effected. The logical solution would be to take the prototype to a job shop where there exists the flexibility necessary to properly work out any outstanding design issues prior to mass manufacture. For this reason we would expect creative design problems to be more easily solved when employing the FFT design method than systematic approaches. Once the problem has been solved (and has become a repetitive design problem) we may consider developing the systematic design solution path that will allow for the rapid completion of subsequent projects.

When considered in this context, it may be readily seen why a solution was found with the FFT method after the project had experienced difficulty under a systematic design method. This situation may be considered ideal though, as the designer was new to the area of design and was receptive to new and radical ideas. Had the designer been familiar with the area of design and/or the project had been a repetitive design problem, the outcome may have been less favourable. It is suggested that the FFT method may not be ideally suited for designers who work with a single preferred design method. It will work well with novice designers or those designers seeking an additional means of completing projects. As with any tool the user must understand under what circumstances it should be used.

\section{Conclusion}

The Familiarization, Functionality and Testing design method allowed for the rapid development of a solution that met customer demands. This design method is well suited to creative design problems as it emphasizes the importance of gathering appropriate background information and investigating the true nature of the design problem prior to beginning embodiment design work. This shift of focus can allow a designer to create solutions more quickly than by systematic methods for creative design as key constraints can be easily overlooked by an overzealous designer.

When the project parameters are not well known, a process that is able to quickly and easily adapt to the project is more likely to be successful than a process that is designed around a single type of problem. If the designer chooses correctly (oftentimes this is purely luck) when applying a systematic approach, they may produce a solution very quickly. If, however, they make a false assumption or misinterpret the project scope or definition, the solution produced will not satisfy the client's criteria and the designer will be forced to retrace the entire process. They will not be armed with new information, simply a solution to cross off their list. This system of trial and error engineering is not an efficient means of developing a solution, as this is a long process where there is no guarantee of success. 


\section{Acknowledgements}

This work supported by a grant from The Whitaker Foundation.

\section{References}

1. Fordyce, W.E. and International Association for the Study of Pain. Task Force on Pain in the Workplace., Back pain in the workplace : management of disability in nonspecific conditions : a report of the Task Force on Pain in the Workplace of the International Association for the Study of Pain. 1995, Seattle: IASP Press. xiii, $75 \mathrm{p}$

2. Deyo, R.A., Low-back pain. Scientific American, 1998. 279(2): p. 48-53

3. Luo, X., et al., Estimates and patterns of direct health care expenditures among individuals with back pain in the United States. Spine, 2004. 29(1): p. 79-86.

4. $\quad$ Dickey, J.P., et al., Relationship between pain and vertebral motion in chronic low-back pain subjects. Clinical

Biomechanics, 2002. 17(5): p. 345-352.

5. Kawchuk, G.N., O.R. Fauvel, and J. Dmowski, Ultrasonic indentation: a procedure for the noninvasive quantification of force-displacement properties of the lumbar spine. $\mathrm{J}$ Manipulative Physiol Ther, 2001. 24(3): p. 149-56.

6. Hawk, C., et al., Preliminary study of the reliability of assessment procedures for indications for chiropractic adjustments of the lumbar spine. J Manipulative Physiol Ther, 1999. 22(6): p. 382-9.

7. Selvik, G., Roentgen stereophotogrammetric analysis. Acta Radiol, 1990. 31(2): p. 113-26.

8. $\quad$ Hickson, I., The Use of Ultrasound in Medicine. 1995.

9. Ziskin, M.C., Basic Physics of Ultrasound, in The Principles and Practice of Ultrasonography in Obstetrics and Gynaecology, A.C. Fleischer, Editor. 1991, Appleton and Lange: Norwalk, CT. p. 1-14

10. Pahl, G.B., W., Engineering Design: A Systematic Approach. 1988, London: Design Council.

11. Johnston, C.R.C., D.J. Fauvel, O.R. Douglas, D. Eggermont, M., Fundamentals of a First-Year Engineering Design and Communication Course: Familiarization, Functionality and Testing. Proceedings of the 2004 American Society for Engineering Education Annual Conference \& Exposition, 2004.

12. Baraniecki, E., Familiarization an approach to multidisciplinary design. 1999. 